\title{
Equivariant Poincaré Series and Monodromy Zeta Functions of Quasihomogeneous Polynomials
}

\author{
by \\ Wolfgang Ebeling and Sabir M. Gusein-Zade
}

\begin{abstract}
In earlier work, the authors described a relation between the Poincaré series and the classical monodromy zeta function corresponding to a quasihomogeneous polynomial. Here we formulate an equivariant version of this relation in terms of the Burnside rings of finite abelian groups and their analogues.
\end{abstract}

2010 Mathematics Subject Classification: Primary 32S40, 13D40, 19A22.

Keywords: group actions, Burnside rings, zeta functions, Poincaré series.

Let $f\left(x_{1}, \ldots, x_{n}\right)$ be a quasihomogeneous polynomial. In [E], [EG1], a relation was described between the Poincaré series $P_{X}(t)$ of the coordinate ring of the hypersurface singularity $X=\{f=0\}$ and the classical monodromy zeta function $\zeta_{f}(t)$ of $f$. The relation involved the so-called Saito duality [S1], [S2]. Namely, in [EG1] it was shown that

$$
P_{X}(t) \cdot \operatorname{Or}_{X}(t)=\widetilde{\zeta}_{f}^{*}(t)
$$

where $\operatorname{Or}_{X}(t)$ is a rational function determined by the orbit types of the natural $\mathbb{C}^{*}$-action on $X$ (see, e.g., [EG2]), $\widetilde{\zeta}_{f}=\zeta_{f}(t) /(1-t)$ is the reduced monodromy zeta function of $f$, and $\widetilde{\zeta}_{f}^{*}(t)$ is the Saito dual of $\widetilde{\zeta}_{f}(t)$ with respect to the quasidegree of the polynomial $f$.

This relation had no intrinsic explanation. It was obtained by computing both sides and comparing the results. In particular, the role of the Saito duality remained unclear. In [EG3], an equivariant version of the Saito duality for a finite

Communicated by M. Kashiwara. Received June 9, 2011. Revised June 21, 2011.

W. Ebeling: Institut für Algebraische Geometrie, Leibniz Universität Hannover, Postfach 6009, D-30060 Hannover, Germany;

e-mail: ebeling@math . uni-hannover.de

S. M. Gusein-Zade: Faculty of Mechanics and Mathematics, Moscow State University, Moscow, GSP-1, 119991, Russia;

e-mail: sabir@mccme.ru

(C) 2012 Research Institute for Mathematical Sciences, Kyoto University. All rights reserved. 
abelian group $G$ was formulated as a transformation between the Burnside rings of $G$ and of the group $G^{*}$ of its characters. Here we use the Burnside rings and their analogues to define equivariant versions of the ingredients of the relation (1) and to give an equivariant analogue of it. This generalization can help to understand the role of the ingredients of the relation, in particular, of the Saito duality.

Let $f$ be a quasihomogeneous polynomial in $n$ variables $x_{1}, \ldots, x_{n}$ of degree $d$ with weights $q_{1}, \ldots, q_{n}\left(q_{i}\right.$ are positive integers, $\left.\operatorname{gcd}\left(q_{1}, \ldots, q_{n}\right)=1\right)$, i.e.

$$
f\left(\lambda^{q_{1}} x_{1}, \ldots, \lambda^{q_{n}} x_{n}\right)=\lambda^{d} f\left(x_{1}, \ldots, x_{n}\right) \quad \text { for } \lambda \in \mathbb{C} .
$$

From now on we assume that $f$ determines the system of weights $\left(q_{1}, \ldots, q_{n} ; d\right)$ in a unique way. This means that in the lattice $\mathbb{Z}^{n}$ of monomials in $x_{1}, \ldots, x_{n}$ (a point $\left(k_{1}, \ldots, k_{n}\right) \in \mathbb{Z}^{n}$ corresponds to the monomial $x_{1}^{k_{1}} \cdots x_{n}^{k_{n}}$ ), the monomials appearing in $f$ with non-zero coefficients generate an affine hyperplane (namely $\left.\left\{\sum_{i} q_{i} k_{i}=d\right\}\right)$. The system of weights $\left(q_{1}, \ldots, q_{n} ; d\right)$ defines a $\mathbb{C}^{*}$-action on the space $\mathbb{C}^{n}$ :

$$
\lambda *\left(x_{1}, \ldots, x_{n}\right)=\left(\lambda^{q_{1}} x_{1}, \ldots, \lambda^{q_{n}} x_{n}\right) .
$$

Let

$$
G_{f}=\left\{\underline{\lambda}=\left(\lambda_{1}, \ldots, \lambda_{n}\right) \in\left(\mathbb{C}^{*}\right)^{n}: f\left(\lambda_{1} x_{1}, \ldots, \lambda_{n} x_{n}\right)=f\left(x_{1}, \ldots, x_{n}\right)\right\}
$$

be the (abelian) symmetry group of $f$, i.e. the group of diagonal linear transformations of $\mathbb{C}^{n}$ preserving $f$. Let

$$
\bar{G}_{f}=\left\{\underline{\lambda} \in\left(\mathbb{C}^{*}\right)^{n}: f\left(\lambda_{1} x_{1}, \ldots, \lambda_{n} x_{n}\right)=\alpha(\underline{\lambda}) f\left(x_{1}, \ldots, x_{n}\right)\right\}
$$

be the extended symmetry group of $f$, i.e. the group of diagonal linear transformations of $\mathbb{C}^{n}$ preserving $f$ up to a constant factor $\left(\alpha: \bar{G}_{f} \rightarrow \mathbb{C}^{*}\right.$ is a one-dimensional representation of the group $\bar{G}_{f}$ ). In other words, this is the group of diagonal linear transformations preserving the hypersurface $X=\{f=0\} \subset \mathbb{C}^{n}$. The group $\bar{G}_{f}$ contains both the symmetry group $G_{f}$ and the group $\mathbb{C}^{*}$ corresponding to the action (2) and is generated by these two subgroups. The intersection of $G_{f}$ and $\mathbb{C}^{*}$ is the cyclic subgroup of order $d$ in $\mathbb{C}^{*}$. (It is generated by the monodromy transformation of $f$ : see below.)

For a group $G$, let $R(G)$ be the ring of complex representations of $G$. As an abelian group, $R(G)$ is freely generated by the isomorphism classes of irreducible representations of $G$. For an abelian group $G$ (say, for a subgroup of $G_{f}$ or of $\bar{G}_{f}$ ) all irreducible representations are one-dimensional, i.e. are elements of the group of characters $G^{*}=\operatorname{Hom}\left(G, \mathbb{C}^{*}\right)$.

Let $\bar{G}$ be a subgroup of the extended symmetry group $\bar{G}_{f}$ of the polynomial $f$ containing the subgroup $\mathbb{C}^{*}$, and let $G=\bar{G} \cap G_{f}$. We shall call an irreducible 
(one-dimensional) representation $\alpha$ of $\bar{G}$ non-positive $(\alpha \leq 0)$ or negative $(\alpha<0)$ if, for $a \in \mathbb{C}^{*} \subset \bar{G}$, one has $\alpha(a)=a^{k}$ with $k$ non-positive or negative respectively. Let $R_{-}(\bar{G})$ be the subring of $R(\bar{G})$ generated by all non-positive representations. The ring $R_{-}(\bar{G})$ contains the ideal $I$ generated by all negative representations of $\bar{G}$. Let $\widehat{R}_{-}(\bar{G})$ be the completion of the $\operatorname{ring} R_{-}(\bar{G})$ with respect to the ideal $I$. Elements of the ring $\widehat{R}_{-}(\bar{G})$ are (formal) sums of the form $\sum_{\alpha \in \bar{G}^{*}, \alpha \leq 0} s_{\alpha}[\alpha]$ with integer coefficients $s_{\alpha}$. Let $\widehat{I} \subset \widehat{R}_{-}(\bar{G})$ be the corresponding completion of the ideal $I$.

One has the following homomorphism (isomorphism) Exp from $\widehat{I}$ regarded as a group with respect to addition to $1+\widehat{I}$ regarded as a group with respect to multiplication:

$$
\operatorname{Exp}\left(\sum_{\alpha \in \bar{G}^{*}, \alpha<0} s_{\alpha}[\alpha]\right)=\prod_{\alpha \in \bar{G}^{*}, \alpha<0}(1-[\alpha])^{-s_{\alpha}} .
$$

The inverse is the homomorphism $\log : 1+\widehat{I} \rightarrow \widehat{I}$ (cf. [GLM]).

Let $A_{X}=\mathbb{C}[x] /(f)$ be the coordinate ring of the zero level set $X=f^{-1}(0)$. The group $\bar{G}$ acts both on $\mathbb{C}[x]$ and on $A_{X}$ by $a * g(x)=g\left(a^{-1} * x\right)$ for $a \in \bar{G}$. This representation is negative on any $\bar{G}$-invariant one-dimensional subspace of $\mathbb{C}[x]$ or of $A_{X}$ (say, on the subspace generated by a monomial) except the one consisting of the constant functions on which it is non-positive. Let $A$ be either $A_{\mathbb{C}^{n}}=\mathbb{C}[x]$ or $A_{X}$. For a (one-dimensional) irreducible representation $\alpha$ of $\bar{G}$, let $A^{\alpha}$ be the corresponding subspace of $A: A^{\alpha}=\{g \in A: a * g=\alpha(a) g$ for $a \in \bar{G}$ \}. For each $\alpha$, the subspace $A^{\alpha}$ is finite-dimensional and is generated by monomials.

Definition 1. The $\bar{G}$-equivariant Poincaré series $P^{\bar{G}}$ of the $\operatorname{ring} A$ is the element of the completion $\widehat{R}_{-}(\bar{G})$ of the representation ring of the group $\bar{G}$ defined by

$$
P^{\bar{G}}=\sum_{\alpha \in \bar{G}^{*}, \alpha \leq 0} \operatorname{dim} A^{\alpha} \cdot[\alpha] .
$$

Remark. For $\bar{G}=\mathbb{C}^{*}$, an irreducible $\mathbb{C}^{*}$-representation is a power of the tautological representation. If one denotes the inverse of the tautological representation by $t$, one gets the Poincaré series (3) as a power series in $t$. In this case it coincides with the usual Poincare series of the ring $A$ corresponding to the (quasihomogeneous) grading defined by the weights.

For $A=A_{X}$ or $A_{\mathbb{C}^{n}}$ we shall denote the $\bar{G}$-equivariant Poincaré series $P^{\bar{G}}$ by $P_{X}^{\bar{G}}$ and $P_{\mathbb{C}^{n}}^{\bar{G}}$ respectively. Let $\alpha_{x_{i}}, i=1, \ldots, n$, and $\alpha_{f}$ be the representations of $\bar{G}$ on the one-dimensional subspaces in $\mathbb{C}[x]$ generated by $x_{i}$ and $f$ respectively. 
Proposition 2.

$$
P_{X}^{\bar{G}}=\frac{1-\left[\alpha_{f}\right]}{\prod_{i=1}^{n}\left(1-\left[\alpha_{x_{i}}\right]\right)} .
$$

Proof. The proof is essentially the same as in the non-equivariant case. One has

$$
P_{\mathbb{C}^{n}}^{\bar{G}}=\sum_{\left(k_{1}, \ldots, k_{n}\right) \in \mathbb{Z}_{\geq 0}^{n}}\left[\alpha_{x_{1}}^{k_{1}} \cdots \alpha_{x_{n}}^{k^{n}}\right]=\frac{1}{\prod_{i=1}^{n}\left(1-\left[\alpha_{x_{i}}\right]\right)} .
$$

One considers the exact sequence

$$
0 \rightarrow A_{\mathbb{C}^{n}} \stackrel{\cdot f}{\rightarrow} A_{\mathbb{C}^{n}} \stackrel{\pi}{\rightarrow} A_{X} \rightarrow 0
$$

The homomorphism $\pi$ maps $A_{\mathbb{C}^{n}}^{\alpha}$ to $A_{X}^{\alpha}$. If $g \in A_{\mathbb{C}^{n}}^{\alpha}$, i.e. $a * g=\alpha(a) g$ for $a \in \bar{G}$, then $a *(f g)=\alpha(a) \alpha_{f}(a) \cdot f g$, i.e. $f g \in A_{\mathbb{C}^{n}}^{\alpha \cdot \alpha_{f}}$. Therefore $\operatorname{dim} A_{X}^{\alpha}=\operatorname{dim} A_{\mathbb{C}^{n}}^{\alpha}-$ $\operatorname{dim} A_{\mathbb{C}^{n}}^{\alpha / \alpha_{f}}$. This yields the statement.

\section{Corollary 3.}

$$
\log P_{X}^{\bar{G}}=\sum_{i=1}^{n}\left[\alpha_{x_{i}}\right]-\left[\alpha_{f}\right]
$$

Note that $\log P_{X}^{\bar{G}}$ is an element of the representation ring $R(\bar{G})$ (more precisely of the subring $R_{-}(\bar{G}) \subset R(\bar{G})$ ), not only of the completion $\widehat{R}_{-}(\bar{G})$.

Now we recall the necessary definitions and facts about Burnside rings of finite groups (for more details see, e.g., [EG3]) and give an appropriate extension of this notion to subgroups of $\bar{G}_{f}$ containing $\mathbb{C}^{*}$.

Let $G$ be a finite group. A $G$-set is a set with an action of $G$. A $G$-set is irreducible if the action of $G$ on it is transitive. Isomorphism classes of irreducible $G$ sets are in one-to-one correspondence with conjugacy classes of subgroups of $G$ : to the conjugacy class containing a subgroup $H \subset G$ one associates the isomorphism class $[G / H]$ of the $G$-set $G / H$. The Grothendieck ring $K_{0}($ f. $G$-sets) of finite $G$-sets (also called the Burnside ring of $G$; see, e.g., $[\mathrm{K}])$ is the (abelian) group generated by the isomorphism classes of finite $G$-sets modulo the relation $[A \amalg B]=[A]+[B]$ for finite $G$-sets $A$ and $B$. Multiplication in $K_{0}$ (f. $G$-sets) is defined by the cartesian product. As an abelian group, $K_{0}$ (f. $G$-sets) is freely generated by the isomorphism classes of irreducible $G$-sets. The element 1 in $K_{0}$ (f. $G$-sets) is represented by the $G$-set consisting of one point (with the trivial $G$-action).

There is a natural homomorphism from $K_{0}$ (f. $G$-sets) to $R(G)$ which sends a $G$-set $X$ to the (vector) space of (complex valued) functions on $X$.

For a subgroup $H \subset G$ there are natural maps $\operatorname{Res}_{H}^{G}: K_{0}($ f. $G$-sets $) \rightarrow$ $K_{0}($ f. $H$-sets $)$ and $\operatorname{Ind}_{H}^{G}: K_{0}$ (f. $H$-sets $) \rightarrow K_{0}$ (f. $G$-sets). The restriction map $\operatorname{Res}_{H}^{G}$ sends a $G$-set $X$ to the same set considered with the $H$-action. The induction map 
$\operatorname{Ind}_{H}^{G}$ sends an $H$-set $X$ to the product $G \times X$ factorized by the natural equivalence: $\left(g_{1}, x_{1}\right) \sim\left(g_{2}, x_{2}\right)$ if there exists $g \in H$ such that $g_{2}=g_{1} g, x_{2}=g^{-1} x_{1}$ with the natural (left) $G$-action. The induction map $\operatorname{Ind}_{H}^{G}$ sends $\left[H / H^{\prime}\right]\left(H^{\prime}\right.$ is a subgroup of $H)$ to $\left[G / H^{\prime}\right]$. Both maps are group homomorphisms, but $\operatorname{Ind}_{H}^{G}$ is not a ring homomorphism.

For an action of a group $G$ on a set $X$ and for $x \in X$, let $G_{x}=\{g \in G$ : $g x=x\}$ be the isotropy group of $x$. For a subgroup $H \subset G$ let $X^{(H)}=\{x \in X$ : $\left.G_{x}=H\right\}$ be the set of points with isotropy group $H$.

We recall the definition of the $G$-equivariant zeta function of $f$ from [EG3]. See an explanation of this notion therein.

The monodromy transformation of $f$ can be defined as the element $h=$ $h_{f} \in G_{f}$ given by

$$
h=\left(\exp \left(2 \pi i q_{1} / d\right), \ldots, \exp \left(2 \pi i q_{n} / d\right)\right) .
$$

As a map from the Milnor fibre $V_{f}=f^{-1}(1)$ to itself, $h$ defines a (faithful) action of the cyclic group $\mathbb{Z}_{d}=\langle h\rangle$ of order $d$ on $V_{f}$. Let

$$
\zeta_{f}(t)=\prod_{q \geq 0}\left(\operatorname{det}\left(\operatorname{id}-t \cdot h_{*} \mid H_{q}\left(V_{f}\right)\right)\right)^{(-1)^{q}}
$$

be the (classical) monodromy zeta function of $f$ (that is, the zeta function of the transformation $h$ ). One can show that in the above-described situation one has

$$
\zeta_{f}(t)=\prod_{m \mid d}\left(1-t^{m}\right)^{s_{m}},
$$

where $s_{m}=\chi\left(V_{f}^{\left(\mathbb{Z}_{d / m}\right)}\right) / m$ are integers. If in (4) one considers the action of $h_{*}$ on the reduced homology groups of $V_{f}$, one obtains the reduced monodromy zeta function $\widetilde{\zeta}_{f}(t)=\zeta_{f}(t) /(1-t)$.

There is a natural one-to-one correspondence between functions of the form

$$
\varphi(t)=\prod_{m \mid d}\left(1-t^{m}\right)^{s_{m}}
$$

and elements of the Burnside ring $K_{0}$ (f. $\mathbb{Z}_{d}$-sets) of the cyclic group $\mathbb{Z}_{d}$ (see [EG3]). The function $\varphi(t)$ from (5) corresponds to the element

$$
\left.\sum_{m \mid \bar{d}} s_{m}\left[\mathbb{Z}_{d} / \mathbb{Z}_{d / m}\right] \in K_{0} \text { (f. } \mathbb{Z}_{d} \text {-sets }\right) .
$$

For the monodromy transformation $h_{f}$ in these terms one has

$$
\zeta_{f}=\sum_{H \subset \mathbb{Z}_{d}} \chi\left(V_{f}^{(H)} / \mathbb{Z}_{d}\right)\left[\mathbb{Z}_{d} / H\right] \in K_{0}\left(\text { f. } \mathbb{Z}_{d} \text {-sets }\right) .
$$


The coefficient $\chi\left(V_{f}^{(H)} / \mathbb{Z}_{d}\right)$ is the Euler characteristic of the space (manifold) of orbits of type $\mathbb{Z}_{d} / H$ in $V_{f}$.

Now let $G$ be a subgroup of the symmetry group $G_{f}$ of the quasihomogeneous polynomial $f$ containing the monodromy transformation $h$. Equation (6) inspires the following definition (see [EG3]).

Definition 4. The G-equivariant zeta function of $f$ is the element

$$
\zeta_{f}^{G}=\sum_{H \subset G} \chi\left(V_{f}^{(H)} / G\right)[G / H]
$$

of the Burnside ring $K_{0}$ (f. $G$-sets).

The coefficient $\chi\left(V_{f}^{(H)} / G\right)$ is the Euler characteristic of the space (manifold) of orbits of type $G / H$ in $V_{f}$.

Definition 5. The reduced G-equivariant zeta function of $f$ is $\widetilde{\zeta}_{f}^{G}=\zeta_{f}^{G}-1$.

Let $K_{0}(\mathrm{f} . \bar{G}$-sets) be the Grothendieck group of $\bar{G}$-sets with finite numbers of orbits and finite isotropy groups of points. This group is freely generated by the classes of the $\bar{G}$-sets $\bar{G} / H$ with finite subgroups $H$.

Remark. There is no natural ring structure on $K_{0}$ (f. $\bar{G}$-sets).

As above (for finite groups), one also has the natural induction map $\operatorname{Ind}_{G}^{\bar{G}}$ : $K_{0}$ (f. $G$-sets) $\rightarrow K_{0}$ (f. $\bar{G}$-sets) which sends $[G / H]$ to $[\bar{G} / H]$ for a subgroup $H \subset G$. A left inverse to this map is the reduction map Red : $K_{0}$ (f. $\bar{G}$-sets) $\rightarrow K_{0}$ (f. $G$-sets) which sends $[\bar{G} / H]$ to $[G / H \cap G](H \subset \bar{G},|H|<\infty)$.

The group $\bar{G}$ acts on the zero level set $X$ of $f$. The $\bar{G}$-equivariant orbit invariant $\operatorname{Or}_{X}^{\bar{G}}$ counts the orbits of the $\bar{G}$-action on $X$ of different types.

Definition 6 (cf. the definition in [EG1], [EG2]).

$$
\operatorname{Or}_{X}^{\bar{G}}:=\sum_{H \subset \bar{G},|H|<\infty} \chi\left(X^{(H)} / \bar{G}\right)[\bar{G} / H] \in K_{0} \text { (f. } \bar{G} \text {-sets). }
$$

Let the tautological map Tau from the ideal $I \subset R_{-}(\bar{G})$ to the group $K_{0}$ (f. $\bar{G}$-sets) be the (additive) group homomorphism mapping the class $[\alpha]$ of a one-dimensional representation $\alpha$ to the class of the punctured space $\mathbb{C}^{*}$ of the space (line) $\mathbb{C}^{1}$ of the representation with the action of $\bar{G}$ defined by $\alpha$. Note that Tau $[\alpha]$ can be defined in the same way for a positive representation $\alpha$ and one has Tau $[\alpha]=\operatorname{Tau}\left[\alpha^{-1}\right]$ (via the isomorphism of $\mathbb{C}^{*}$ with itself which sends $z \in \mathbb{C}^{*}$ to $\left.z^{-1}\right)$. 
Theorem 7. One has

$$
\operatorname{Tau}\left(\log P_{X}^{\bar{G}}\right)-\operatorname{Or}_{X}^{\bar{G}}=\operatorname{Ind}_{G}^{\bar{G}} \widetilde{\zeta}_{f}^{G}
$$

in $K_{0}(f \cdot \bar{G}$-sets $)$.

Proof. For $I \subset I_{0}=\{1, \ldots, n\}$, let $|I|$ be the number of elements of $I$, let $\left(\mathbb{C}^{*}\right)^{I}:=$ $\left\{\left(x_{1}, \ldots, x_{n}\right) \in \mathbb{C}^{n}: x_{i} \neq 0\right.$ for $i \in I, x_{i}=0$ for $\left.i \notin I\right\}$ be the corresponding coordinate torus of dimension $|I|\left(\left(\mathbb{C}^{*}\right)^{\emptyset}=\emptyset\right)$, and let $G^{I} \subset \bar{G}$ be the isotropy subgroup $\left\{a \in \bar{G}: a x=x\right.$ for all $\left.x \in\left(\mathbb{C}^{*}\right)^{I}\right\}$. Let $X^{I}=X \cap\left(\mathbb{C}^{*}\right)^{I}$ and $Y^{I}=X^{I} / \bar{G}$. One has

$$
\operatorname{Or}_{X}^{\bar{G}}=\sum_{I} \chi\left(Y^{I}\right) \cdot\left[\bar{G} / G^{I}\right]
$$

The Milnor fibre $V_{f}=f^{-1}(1)$ is the union $\bigcup_{I}\left(V_{f} \cap\left(\mathbb{C}^{*}\right)^{I}\right)$ of $G$-invariant varieties. Therefore

$$
\widetilde{\zeta}_{f}^{G}=\sum_{I} \chi\left(\left(V_{f} \cap\left(\mathbb{C}^{*}\right)^{I}\right) / G\right)\left[G / G^{I}\right]-1=\sum_{I}\left[\chi\left(\left(\mathbb{C}^{*}\right)^{I} / \bar{G}\right)-\chi\left(Y^{I}\right)\right]\left[G / G^{I}\right]-1 .
$$

Note that, if $G^{I} \not \subset G$, then $V_{f} \cap\left(\mathbb{C}^{*}\right)^{I}=\emptyset$. For $|I| \neq 1$, one has $\chi\left(\left(\mathbb{C}^{*}\right)^{I} / \bar{G}\right)=0$, and for $|I|=1, \chi\left(\left(\mathbb{C}^{*}\right)^{I} / \bar{G}\right)=1$. Therefore

$$
\operatorname{Or}_{X}^{\bar{G}}+\operatorname{Ind}_{G}^{\bar{G}} \widetilde{\zeta}_{f}^{G}=\sum_{i=1}^{n}\left[\bar{G} / G^{\{i\}}\right]-\operatorname{Ind}_{G}^{\bar{G}} 1=\operatorname{Tau}\left(\log P_{X}^{\bar{G}}\right) .
$$

Remark. 1. One could prefer to have an equation like (8) in the Burnside ring $K_{0}$ (f. $G$-sets). One can see that (8) implies the equation

$$
\widetilde{\zeta}_{f}^{G}=\operatorname{Red}\left(\operatorname{Tau}\left(\log P_{X}^{\bar{G}}\right)-\operatorname{Or}_{X}^{\bar{G}}\right)
$$

in $K_{0}$ (f. $G$-sets) with the reduction map Red : $K_{0}$ (f. $\bar{G}$-sets $) \rightarrow K_{0}$ (f. $G$-sets). However from a formal point of view (9) is weaker than (8).

2. Looking at the relation (8), one observes that there is no Saito duality in the sense of [EG3] involved in it. It appears in (1) because of the method used to encode the $\mathbb{C}^{*}$-action on $X$ in $\operatorname{Or}_{X}(t)$.

3. One can see that, generally speaking, both $\operatorname{Or}_{X}^{\bar{G}}$ and $\widetilde{\zeta}_{f}^{G}$ contain much more summands than $\operatorname{Tau}\left(\log P_{X}^{\bar{G}}\right)$. In particular, $\operatorname{Tau}\left(\log P_{X}^{\bar{G}}\right)$ contains only summands represented by irreducible $\bar{G}$-sets isomorphic (as varieties) to $\mathbb{C}^{*}$. This gives the hint that (8) (and therefore also (1)) is essentially a relation between $\operatorname{Or}_{X}^{\bar{G}}$ and $\widetilde{\zeta}_{f}^{G}$, where the Poincaré series $P_{X}^{\bar{G}}$ plays rather the role of a correction term. 


\section{Acknowledgements}

This research was partly supported by the DFG Mercator program (INST 187/ 490-1), the Russian government grant 11.G34.31.0005, RFBR-10-01-00678, NSh-4850.2012.1 and Simons-IUM fellowship.

\section{References}

[E] W. Ebeling, Poincaré series and monodromy of a two-dimensional quasihomogeneous hypersurface singularity, Manuscripta Math. 107 (2002), 271-282. Zbl 1036.14017 MR 1906197

[EG1] W. Ebeling and S. M. Gusein-Zade, Poincaré series and zeta function of the monodromy of a quasihomogeneous singularity, Math. Res. Lett. 9 (2002), 509-513. Zbl 1056.14003 MR 1928870

[EG2] Lectures on monodromy, in Singularities in geometry and topology, World Sci., Hackensack, NJ, 2007, 234-252. Zbl 1135.32029 MR 2311489

[EG3] Saito duality between Burnside rings for invertible polynomials, Bull. London Math. Soc. 44 (2012), 814-822.

[GLM] S. M. Gusein-Zade, I. Luengo and A. Melle-Hernández, A power structure over the Grothendieck ring of varieties, Math. Res. Lett. 11 (2004), 49-57. Zbl 1063.14026 MR 2046199

[K] D. Knutson, $\lambda$-rings and the representation theory of the symmetric group, Lecture Notes in Math. 308, Springer, Berlin, 1973. Zbl 0272.20008 MR 0364425

[S1] K. Saito, Duality for regular systems of weights: a précis, in Topological field theory, primitive forms and related topics, M. Kashiwara et al. (eds.), Progr. Math. 160, Birkhäuser, Boston, 1998, 379-426. Zbl 1058.32502 MR 1653033

[S2] _ Duality for regular systems of weights, Asian J. Math. 2 (1998), 983-1047. Zbl 0963.32023 MR 1734136 